\title{
KSK zu TarMed: Taxpunktwert unter einem Franken
}

Der neue Arzttarif TarMed muss kostenneutral eingeführt werden. KSK-Berechnungen zeigen, dass deshalb der Starttaxpunktwert in allen Kantonen deutlich unter einen Franken zu liegen kommt.

Versicherer und Versicherte erwarten, dass mit der Einführung des neuen Arzttarifs TarMed keine Kostensteigerung verbunden ist. Entscheidend für die Frage der Kostenneutralität wird die Festlegung der kantonalen Taxpunktwerte (TPW) sein.

Weil für das KSK die kostenneutrale Einführung des neuen Arzttarifs oberste Priorität besitzt, ist es derzeit dabei, aufgrund einer Leistungskorbstudie für sämtliche Kantone Berechnungen zum Starttaxpunktwert durchzuführen. Dies geschieht in Zusammenarbeit mit Leistungsexperten der Versicherer und der Kantonalverbände. Erste provisorische Ergebnisse liegen vor; sie zeigen klar: In allen Kantonen sollte nach den Berechnungen des KSK der Starttaxpunktwert für den Bereich der Arztpraxen deutlich unter einen Franken zu liegen kommen. Selbst in den teuersten Kantonen wie Genf und Basel-Stadt wird der Wert von einem Franken deutlich unterschritten.

Dass die Leistungskorbstudie des KSK plausible Ergebnisse liefert, zeigt das Beispiel einer vom Kanton Wallis Ende Februar publizierten Studie. Dort liegt der vom Kanton, den Versicherern und dem Walliser Ärzteverband gemeinsam berechnete Starttaxpunktwert bei 72 Rappen. Das entspricht in etwa den Berechnungen des KSK.

Das KSK geht davon aus, dass nur mit Werten in der Grössenordnung, wie sie die Studien des KSK und des Kantons Wallis ermittelt haben, die kostenneutrale Einführung des TarMed sichergestellt werden kann. Es fordert zudem, dass die wichtigsten Eckwerte der Tarifstruktur zum Zeitpunkt der Einführung des Tarifs nicht zu hoch bewertet werden. Mit dieser Ausgangslage ist die Kostenneutralität einfacher zu erreichen als durch eine spätere Anpassung der kantonalen Taxpunktwerte.

Das Konkordat erwartet im übrigen, dass der Starttaxpunktwert für die Eidgenössischen Sozialversicherer (Unfallversicherer, IV, MV), der gesamtschweizerisch festgelegt wird, ebenfalls deutlich unter einen Franken zu liegen kommt, damit die Kostenneutralität nicht zum Nachteil der Krankenversicherer gefährdet wird.

\section{Pressecommuniqué FMH zu KSK}

Das Konkordat der Schweizer Krankenversicherer hat mit Datum vom 21. März 2000 ein Pressecommuniqué zur Einführung der TarMed-Tarifstruktur in den kantonalen Kassenverträgen publiziert. Dieses gipfelt in der Behauptung, der Starttaxpunktwert der Tarife müsse, selbst in den teuersten Kantonen Basel und Genf, deutlich unter einem Franken liegen.

Die Verbindung der Schweizer Ärztinnen und Ärzte FMH hält dazu folgendes fest:

1. Die Verhandlungen bezüglich der Modalitäten zur Festlegung des Start-Taxpunktwertes wie auch der Kostenneutralität waren bisher im Gange; die FMH wird zu entscheiden haben, wie sie mit dieser Stellungnahme des KSK «avant la lettre» umgehen will.

2. Die vom KSK herbeigezogene Leistungskorbstudie kann im besten Falle als Parteienmeinung eingestuft werden; weder Vorgehensweisen noch Resultate sind in irgendeiner Art und Weise validiert oder gar akzeptiert.

3. Die vom KSK zitierte Walliser Studie ist nachgewiesenermassen mit schweren Mängeln behaftet:

- Sie basiert auf Daten, die aufgrund einer veralteten Version der TarMed-Tarifstruktur (Version Beta 3), also noch vor Umsetzung der preisüberwacherlichen Empfehlungen(!) vorgelegen hat.

- Sie wurde ausschliesslich in einem Sample schwergewichtig von Grundversorgern durchgeführt und kann deshalb in keiner Weise als repräsentativ bezeichnet werden.

4. Die FMH wird in Kürze die massgeblichen Vorgehensweisen und Daten, die einer kostenneutralen Einführung der Tarife dienen könnten, in objektiver und seriöser Art publizieren.

\section{Communiqué de presse de la FMH à propos du CAMS}

Dans son communiqué de presse du 21 mars 2000 sur l'introduction de la structure tarifaire dans les conventions tarifaires cantonales, le Concordat des assureurs-maladie suisses (CAMS) affirme que la valeur du point tarifaire devrait se trouver nettement sous la barre du franc, même pour les cantons les plus chers de Bâle et de Genève.

Ce communiqué appelle de la part de la Fédération des médecins suisse (FMH) les remarques suivantes:

1. Les modalités de calcul de la valeur initiale du point et la question de la neutralité des coûts font partie des négociations en cours; la FMH s'interroge par conséquent sur la suite à donner à cette prise de position du CAMS avant la lettre.

2. L'étude des paniers de prestations mentionnée par le CAMS constitue au mieux un parti pris; ni la méthode utilisée ni les résultats n'ont été validés ou acceptés d'une quelconque manière.

3. Quant à l'étude menée en Valais et citée par le CAMS, elle présente de graves lacunes comme on peut aisément le démontrer:

- Elle se fonde sur des données tirées d'une ancienne version de la structure tarifaire TarMed (version bêta 3), antérieure à la mise en application des recommandations de M. Prix (!).

- Elle a porté sur un échantillon composé principalement de médecins de premier recours et n'est donc en aucune manière représentative.

4. La FMH publiera très prochainement un texte objectif et sérieux sur les méthodes et données essentielles dont on pourrait se servir pour introduire les tarifs en maintenant le statu quo sur l'ensemble des coûts. 